Background and Aims Advantages of PCEA over CEA have been demonstrated in obstetric patients. Whether similar benefit applies to surgical patients is unclear.

Aim: To assess possible advantages of patient controlled epidural analgesia (PCEA) over continuous epidural analgesia (CEA) in surgical patients

Methods Embase, PubMed and Cochrane library were searched, enabling systematic review of studies comparing PCEA and CEA in adult surgical patients (PROSPERO: CRD42018106644). Study quality was assessed using Cochrane Risk-of-Bias tool (RoB2). Primary outcome: pain score on postoperative day one (POD1). Secondary outcomes: 24 or 48 hour epidural or intravenous total analgesic dose, manual top-ups and patient satisfaction

Results Eleven trials (ten RCTs, one cohort-analysis, 1687 patients) with high heterogeneity of study characteristics were identified with a high to intermediate risk of bias. Three studies showed reduced pain scores on POD1 in PCEA compared to CEA patients $(36-42 \%, \mathrm{P}<0.05)$. Seven studies found comparable pain scores between groups, one study a higher pain score in PCEA patients. PCEA-use reduced epidural medication $(28 \%$ to $76 \%$ reduction, $\mathrm{P}<0.01)$ in seven studies. Two studies found lower top-up frequency and higher analgesic satisfaction in PCEA; PCEA patients used less intravenous morphine $(0.16$ vs $3.45 \mathrm{mg}$ per patient, $\mathrm{P}<0.05$ ) in one study.

Conclusions Regarding pain scores, rescue systemic analgesics and patient satisfaction, PCEA in surgical patients had limited advantages over CEA. PCEA reduced the amount of epidural medication and top-up frequency. On the basis of current available evidence, we cannot conclude that PCEA offers major benefits over CEA in surgical patients.

\section{IMPACT OF THE ERECTOR SPINAE PLANE BLOCK ON THE POSTOPERATIVE PAIN OF LUMBAR SPINAL STENOSIS SURGERY. A SINGLE BLIND RCT- A 70\% PATIENT'S INTERIM ANALYSIS}

${ }^{1} \mathrm{~S}$ Van Migem*, ${ }^{1} \mathrm{~N}$ Parisi, ${ }^{1} \mathrm{~L}$ Van Der Essen, ${ }^{1} \mathrm{~V}$ Marneffe, ${ }^{2} \mathrm{H}-\mathrm{M}$ Dehbi, ${ }^{1} \mathrm{G}$ Samouri. ${ }^{1}$ Clinique Saint-Pierre Ottignies, Ottignies-Louvain-la-Neuve, Belgium; ${ }^{2}$ UCL, London, UK

\subsection{6/rapm-2021-ESRA.221}

Background and Aims The erector spinae plane (ESP) block was described in 2016 by Forero et al. It involves the injection of local anesthetic into the interfascial plane, deep to erector spinae muscle, allowing the blockade of the dorsal and ventral rami of the thoracic spinal nerves. It was initially proposed for analgesia of costal fractures, pulmonary lobectomy and thoracic vertebrae. The ESP block (ESPB) could probably be extended to a large number of surgical procedures.

Methods After ethical commitee approval and informed consent, 80 patients were included in this prospective, single blind, monocentric RCT for lombar spinal stenosis surgery (LSSS) under general anesthesia: 40 patients with ESPB, 40 patients with local infiltration (LI) by the surgeon. The current interim reporting is based on 28 patients in ESPB and 28 in LI. Piritramide consumption was followed. The ESPB was realized on T12 and ultrasound-guided (chirocaine $0.25 \%+$ epinephrine 1:200.000 $4 \mathrm{mg} / \mathrm{kg}$ ). The control group was injected at the same concentration by the surgeon. Complementary analgesia was realized with Patient Controlled Analgesia (Piritramide), paracetamol and ketorolac.
Results After performing a T-test to compare the means of piritramide consumption at day 1 , we did not find any significant difference between the 2 groups (ESPB $12.9 \mathrm{mg}$ versus LI $14.7 \mathrm{mg}, \mathrm{p}=0.55)$. A Mann-Withney U-test was also performed and did not show any difference.

Conclusions After collecting data from $70 \%$ of the population, we cannot conclude that there is a benefit of ESPB over LI by the surgeon.

\section{THE USE OF REGIONAL ANAESTHESIA AND IDENTIFYING COMPARTMENT SYNDROME: A CASE REPORT}

C Hopkins*, C Broe, B Bellew. Imperial College Healthcare NHS Trust, London, UK

\subsection{6/rapm-2021-ESRA.222}

Background and Aims Acute compartment syndrome is a surgical emergency, and must be identified promptly in order to limit potential complications. The use of regional anaesthesia remains controversial for patients who are at higher risk of developing compartment syndrome due to concerns that the cardinal symptoms may be masked, and diagnosis subsequently delayed. This is a case report of a clinical situation which opposes this theory.

Methods A 31 year old male presented to a major trauma centre following a road traffic accident. Radiological imaging showed he had sustained multiple injuries, including a closed right tibia and fibula fracture and an extensive left calf laceration which required operative management. A lumbar epidural was inserted for intra-operative and postoperative analgesia, prior to the patient undergoing IM nail fixation of the right tibial fracture and exploration of the left leg laceration under general anaesthesia.

Results The lumbar epidural provided good analgesia which was maintained with a continuous infusion of $0.125 \%$ levobupivacaine and $2 \mathrm{mcg} / \mathrm{ml}$ fentanyl. Fourteen hours postoperatively, this patient developed severe right lower limb pain. Intra-compartmental pressures were measured at this stage, where it was noted that anterior compartment pressures were abnormal and the patient underwent urgent fasciotomy under general anaesthesia. Operative findings at this time were consistent with a diagnosis of compartment syndrome, with maintained viable muscle tissue present on examination.

Conclusions This case demonstrates support for alternative theories that regional anaesthetic techniques using low concentrations may provide effective analgesia without the risk of masking the presentation of acute compartment syndrome.

\section{POST OPERATIVE PAIN MANAGEMENT FOR HIP FRACTURE SURGERY, A NEW PROTOCOL EVALUATION}

${ }^{1,2} \mathrm{H} \mathrm{Ahmed}^{*},{ }^{3} \mathrm{G}$ Deloughry, ${ }^{3} \mathrm{~K}$ Joyce, ${ }^{3} \mathrm{~B} \mathrm{O}^{\prime}$ Donnell, ${ }^{3} \mathrm{~F}$ Loughnane. ${ }^{1}$ Faculty of Medicine, Cairo University, Cairo, Egypt; ${ }^{2}$ Leeds Teaching Hospitals, Leeds, UK; ${ }^{3}$ Cork University Hospital, Cork, Ireland

\subsection{6/rapm-2021-ESRA.223}

Background and Aims Hip fracture surgeries represent a significant burden on the healthcare system. Delirium could affect up to $61 \%$ of patients with hip fracture, and is associated with delayed recovery, higher morbidity and poor cognitive function. $^{(1)}$ 